\title{
A modular, partitioned, discrete element framework for industrial grain distribution systems with rotating machinery
}

\author{
Guillermo Casas $^{1} \cdot$ Debanjan Mukherjee $^{2} \cdot$ Miguel Angel Celigueta $^{1} \cdot$ \\ Tarek I. Zohdi ${ }^{2}$. Eugenio Onate ${ }^{1}$
}

\begin{abstract}
A modular discrete element framework is presented for large-scale simulations of industrial grain-handling systems. Our framework enables us to simulate a markedly larger number of particles than previous studies, thereby allowing for efficient and more realistic process simulations. This is achieved by partitioning the particle dynamics into distinct regimes based on their contact interactions, and integrating them using different time-steps, while exchanging phase-space data between them. The framework is illustrated using numerical experiments based on fertilizer spreader applications. The model predictions show very good qualitative and quantitative agreement with available experimental data. Valuable insights are developed regarding the role of lift vs drag forces on the particle trajectories in-flight, and on the role of geometric discretization errors for surface meshing in governing the emergent behavior of a system of particles.
\end{abstract}

Guillermo Casas and Debanjan Mukherjee have contributed equally to this work.

\footnotetext{
Debanjan Mukherjee

debanjan@berkeley.edu

Guillermo Casas

gcasas@cimne.upc.edu

Miguel Angel Celigueta

maceli@cimne.upc.edu

Tarek I. Zohdi

zohdi@berkeley.edu

Eugenio Onate

onate@cimne.upc.edu

1 International Center for Numerical Methods in Engineering (CIMNE), Universitat Politecnica de Catalunya, Barcelona, Spain

2 Department of Mechanical Engineering, University of California, Berkeley, USA
}

Keywords Discrete element method · Contact · Grain distribution $\cdot$ Modular simulations $\cdot$ Rotary spreaders

\section{Introduction}

The handling of particles and grains in industrial facilities is an issue of great importance. Grains and particulate media comprise perhaps the most widely handled and manipulated material after water, across a wide range of industrial applications [13]. These include handling and processing of pharmaceutical materials [33], agricultural and food grain processing [42], and handling of minerals [5,31]. Particulate media processing is increasingly receiving extensive attention in advanced manufacturing applications as well $[51,52]$. Improved understanding of the processes and mechanisms of grain handling will therefore be greatly beneficial towards better design and operation of such systems. In this work, we present a computer simulation framework based on the discrete element method, to create numerical models for industrial grain-handling systems. The focus is on grain distribution systems, wherein typically large volumes of grains are introduced onto a rotating, conveying, machinery, and are then dispersed across a region in space within the handling facility or in the open.

The discrete element method (DEM) provides an ideal computational tool for the numerical simulation of such processes, since individual grain dynamics can be captured, and emergent properties from the grain motion can be easily tracked. However, for realistic process simulations, oftentimes really large number of particles are required to be modeled. This renders such computations inordinately expensive. The computational expense is further governed by the numerical integration time-step intervals used for computing the particle positions and velocities. Based on their 
interactions with each other, a collection of particles can either be categorized as (a) sparsely interacting or dilute, with rare inter-particle contact occurrence, (b) collisionally driven, where inter-particle contacts occur over durations much smaller than relevant physical time-scales, or, (c) particles in enduring contact, where large contact durations are observed. The resolution of the contact events places a restriction on the numerical time-step size, such that integration occurs over time-steps that resolve the contact duration. This restriction affects simulation time, as well as computational expense, significantly.

DEM-based computational models have received an increasing extent of attention for grain-handling simulations across multiple industries. Simulation of grains in tumbling ball-mills has been studied extensively by [4], and [29,30] amongst others and is a relevant problem for mineral and ore processing. It is a classic example of grain handling using rotating machinery. For the pharmaceutical industry, an overview of DEM for process modeling simulations can be found in [23]. Detailed studies on specifics of model development for contact-driven particle dynamics has been addressed more recently by [26], and a recent example of more generalized process and operation modeling for pharmaceutical industry using DEM can be found in [1]. Particulate and powder handling is an important component for a range of additive manufacturing processes like selective laser sintering (see [10,49]), powder injection molding [50], and spray deposition processes [32]. In various forms, these applications involve processes that transport large volumes of particles via moving, mostly rotating, machinery. While for most of the applications cited here, this transport and distribution process has been relatively less studied, there exist a substantial number of studies on this aspect in the area of agricultural grains and fertilizer handling. The theoretical analysis of the motion of particles on rotating disks with vanes can be traced back in time, at least, to the work presented by [36], who gave an expression applicable to conical disks with radial vanes and another for pitched vanes on a flat disk (see [12]). Successive improvements have been (and are still being) proposed to generalize this work (see for example [12,48] and [7]). These semi-empirical approaches can only take into account the trajectories of isolated particles, often under specific restrictions (for example, not allowing bouncing). As in many other areas of handling of granular matter, the recent increase in computational power has allowed the effective application of the DEM to this field, in which large particle numbers in above the thousands commonly occur. Despite this fact, only a small number of studies have been reported (see [47] for a relatively recent review on the subject). In [44] the authors compare single-particle DEM results with the semi-empirical models, showing their deficiencies, and further extend their work in [45], using a different spring-dashpot model than the one proposed in the present work. These studies compared DEM simulations of two types of spreaders and reported good qualitative agreement with experimental data (see also [47]). In [46], the authors presented a sensitivity analyses of a conical spreader with respect to its physical systems using the same DEM framework as in [45]. In another study by [6], a linear springdashpot model was used and the systems physical parameters were calibrated to match experimental data on-ground spread patterns.

Given the broad applicability of DEM models for such processes, their essential complications, and their industrial relevance, it is valuable to devise simulation frameworks that are specifically aimed to increase numerical efficiency, and tackle the computational costs for handling large volumes of grains. Particularly for grain distribution systems, this can be achieved by taking advantage of the underlying physical interactions and time-scales that the grains experience. During transport via rotating disks, spreaders, and conveyors, it is often found that the particles undergo extended duration of contact-driven dynamics, and thereafter they disperse out, with substantially less inter-particle interactions. It is our objective to devise a specific DEM simulation framework, that targets such applications, and that is more efficient by using a modular approach to partition the particle dynamics into distinct regimes. Our presentation here addresses not only model development in sufficient detail, but also provides illustrative numerical examples, and benchmarks against available experimental data. All numerical examples are based on the application of agricultural fertilizer spreading using rotating disk spreaders. This choice of application was guided by the availability of sufficient amount of existing literature and data to benchmark our simulation and the predicted trends (qualitative as well as quantitative) against.

To this end, all model details have been extensively addressed in Sect. 2, followed by a detailed discussion of the overall simulation framework, and some implementation details in Sect. 3. Numerical examples, and analysis have been discussed in Sect. 4, with attention to both 'on-disk' and 'in-flight' dynamics of grains, and concluding remarks are presented in Sect. 5, along with comments on ongoing research and future extensions.

\section{Model formulation}

\subsection{Contact model for grain dynamics}

The grain dynamics on the rotating machinery surface is governed by grain-grain, and grain-surface contact interactions. Contact interactions in DEM are modeled using either a soft-sphere approach, where a force-deformation relation is employed, or a hard-sphere approach, where a kinematic, collision-driven velocity modification is employed. While 
both approaches have their unique features, we have chosen here a spring-dashpot type soft-sphere approach.

Considering two spheres in contact, whose centers are located at $\mathbf{r}_{1}$ and $\mathbf{r}_{2}$, respectively, a normal vector for the contacting pair can be defined as follows:

$\mathbf{n}_{21}=\frac{\mathbf{r}_{2}-\mathbf{r}_{1}}{\left\|\mathbf{r}_{2}-\mathbf{r}_{1}\right\|}, \quad \mathbf{n}_{12}=-\mathbf{n}_{21}$

The total contact force can now be defined as the sum of a normal and a tangential force:

$\mathbf{F}=F_{n} \mathbf{n}+F_{t} \mathbf{t}$

where the subscripts 21 and 12 identifying the particles have been omitted for the sake of brevity. The unit vector defining tangential contact interactions $\mathbf{t}$ depends on the tangential force and will be specified in the following.

The normal contact force $F_{n}$ is computed as the combination of an elastic contribution and a viscous damping contribution.

$F_{n}=F_{n, \mathrm{el}}+F_{n, \mathrm{damp}}$

where the elastic contribution $F_{n \text {,el }}$ is a function of the deformation in the corresponding direction, whereas the viscous damping contribution $F_{n \text {,damp }}$ is a function of the velocity of deformation. The corresponding normal deformation or indentation is defined based on a geometric overlap based on the particle positions and their radii as follows:

$\delta_{n}=R_{1}+R_{2}-\left\|\mathbf{r}_{21}\right\|$,

where $R_{1}$ and $R_{2}$ are the radii of contacting particles 1 and 2 , and the value of $F_{n}$ will thus depend on $\delta_{n}$ and its time derivative.

On the other hand, the tangential deformations and velocities will not in general align. Furthermore, the tangential component must not exceed, in modulus, the limit imposed by the Coulomb friction law. Thus, defining $\mathbf{F}_{t}$ as the sum of the tangential elastic and viscous contributions:

$\mathbf{F}_{t}=F_{t, \mathrm{el}} \mathbf{t}_{d}+F_{t, \mathrm{damp}} \mathbf{t}_{v}$

one then takes $\mathbf{t}=\left(\left\|\mathbf{F}_{t}\right\|\right)^{-1} \mathbf{F}_{t}$ and

$F_{t}=\min \left(\frac{\mu F_{n}}{\left|F_{t}\right|}, 1\right) F_{t}$,

where $F_{t}$ denotes the magnitude of $\mathbf{F}_{t}$ and $\mu$ the friction coefficient (we assume no difference exists between static and dynamic friction coefficients). Note here that we do not allow for negative vales of $F_{n}$ (i.e., attractive forces), so there is no need for taking its absolute value in 6 .
The directions $\mathbf{t}_{d}$ and $\mathbf{t}_{v}$ can be explicitly formulated based on considerations of the kinematics during tangential deformation. For this, we assume that under no-slip conditions, the total tangential displacements caused by local deformations can be obtained by integrating the tangential components of the (rigid body motion) velocity of each particle at the point of contact, neglecting spatial differences in the velocity fields between points close to it. Following this, the tangential ana$\log$ to the normal indentation is determined by the tangential component of the relative displacement at the point of contact. Its evolution is described by the expression

$\mathbf{S}(\tau)=\int_{\tau_{0}}^{\tau} \mathbf{u}_{t}(s) \mathrm{d} s+\mathbf{S}_{0}$,

where $\tau_{0}$ is the time at which slip last occurred, and $\mathbf{S}_{0}$ is the residual tangential displacement, possibly non-zero in cases of uninterrupted contact, when prior to slippage, a certain amount of tangential strain is generated, not all of which can be dissipated after the slippage occurs, due to static friction. In this work the value of $\mathbf{S}_{0}$ is set to the value of $\mathbf{S}$ previous to slip (no instantaneous dissipation of elastic energy). The tangential component of the relative velocity at the point of contact is denoted by $\mathbf{u}_{t}$. The overall relative velocity at the point of contact can be defined as follows:

$\mathbf{u}=\dot{\mathbf{r}}_{21}+l_{1} \mathbf{n} \times \omega_{1}-l_{2} \mathbf{n} \times \omega_{2}$

and the corresponding normal and tangential components can then be defined as follows:

$\mathbf{u}_{n}=(\mathbf{u} \cdot \mathbf{n}) \mathbf{n}, \quad \mathbf{u}_{t}=\mathbf{u}-\mathbf{u}_{n}$,

where $\omega_{1}$ and $\omega_{2}$ are the angular velocity vectors of particles 1 and 2 , respectively. The parameters $l_{1}$ and $l_{2}$ denote the lever arms for evaluating the contact moments for the already deformed spheres. From Hertz's theory of contact, it can be shown (see for example [22]) that the displacements at the surface of the spheres in the contact area are proportional to the contact pressures, and to the factor $(1-v) / E$. The share of local maximum indentation taken up by particle $i$ is thus given by the ratio $E^{*} / E_{i}(i=1,2)$. Thus, we assume the following expression for the position of the point of contact, relative to particle 1 (which establishes the lever arm for moment estimation):

$l_{1}=R_{1}-\delta_{1}=R_{1}-\frac{E_{1}}{E^{*}} \delta_{n}$.

Furthermore, for any non-zero tangential displacement we can define the displacement-tangential vector $\mathbf{t}_{d}$ as follows:

$\mathbf{t}_{d}(\tau)=-(\|\mathbf{S}(\tau)\|)^{-1} \mathbf{S}(\tau)$. 
Similarly, for any non-zero tangential velocity we can define the velocity-tangential vector $\mathbf{t}_{v}$ as follows:

$\mathbf{t}_{v}(\tau)=-\left(\left\|\mathbf{u}_{t}(\tau)\right\|\right)^{-1} \mathbf{u}_{t}(\tau)$.

The tangential analog to the indentation between the contacting particles can then be defined as

$\delta_{t}:=\|\mathbf{S}\|$.

Remark In the event of slip, it can be assumed that a part of the relative motion is being accumulated as tangential deformation. This possibility can be accounted for by a factor $\beta$ (say) defined as the proportion of the total tangential velocity due to local tangential deformation. Equation 7 is then generalized to

$\mathbf{S}(\tau)=\int_{\tau_{0}}^{\tau} \beta \mathbf{u}_{t}(s) \mathrm{d} s+\mathbf{S}_{0}$

$\beta$ can be estimated using the fact that during slip the tangential force has a constant modulus, given by the friction law. This consideration leads to a differential relation between the tangential displacements and their derivatives that may be used to numerically calculate the value of $\beta$ at every timestep. For the framework developed here, we have assumed $\beta=1$ (though shortening vector $\mathbf{S}$ to to the maximum elongation that can be held by friction in stationary conditions whenever the modulus of the right-hand side of 14 exceeds this value, that is, disregarding the tangential viscous contribution).

Assuming furthermore that the normal contact forces generate no moment around the point of contact, the estimation of the contact moment based on the tangential contact forces can be formulated as follows:

$\mathbf{M}=F_{t} l_{1} \mathbf{t} \times \mathbf{n}$

where again, the particle subscripts have been omitted for brevity.

Having addressed the kinematics, the expressions for the normal force as function of the normal displacement and relative velocity can now be outlined. We assume here that the elastic component of the force is given by the classical Hertzian model as follows:

$F_{e l, n}=\frac{4}{3} R^{* 1 / 2} E^{*} \delta_{n}^{3 / 2}$,

where $R^{*}:=\left(1 / R_{1}+1 / R_{2}\right)^{-1}, E_{i}^{*}:=E_{i} /\left(1-v_{i}^{2}\right)$, $E^{*}:=\left(1 / E_{1}^{*}+1 / E_{2}^{*}\right)^{-1}\left(E_{i}\right.$ and $v_{i}$ are particle i's Young's moduli and Poisson's ratios, respectively). The corresponding viscous damping contribution is modeled as follows:

$F_{n, \text { damp }}=c_{n} \delta_{n}^{1 / 4} \dot{\delta}_{n}$

The expression for the damping coefficient in particle-wall contact is derived from the formulation presented in [41], and we modify the original expression for particle-wall contact, by using equivalent parameters for the particle-particle contact, as follows:

$c_{n}=\gamma \sqrt{8 E^{*} M^{*} \sqrt{R^{*}}}$,

where $M^{*}$ is $\left(1 / m_{1}+1 / m_{2}\right)^{-1}$ and $\gamma$ is a viscous damping coefficient, which can be estimated based on considerations of energy loss and restitution as described in the next section.

The computation of the tangential component is somewhat more involved, since it is necessarily path-dependent. The formulation presented here was proposed in [41], where however the introduced formulation was already discretized in time. Based on it, we provide the expression for the derivative of $F_{t}$ (see Appendix 2 for some additional details), which must be integrated in time to obtain the value of the force.

$\dot{F}_{t, \text { el }}= \begin{cases}k_{t} \dot{\delta_{t}} & \text { if } \dot{F}_{n} \geq 0 \\ k_{t} \dot{\delta}_{t}+\frac{F_{t, \text { el }} \dot{k_{t}}}{k_{t}} & \text { if } \dot{F}_{n}<0,\end{cases}$

where $k_{t}=8 G^{*} \sqrt{R \delta_{n}}$ and $G^{*}=G /(4-2 v)$, with $G=$ $E /(2+2 v)$. This expression can alternatively be expressed as

$\dot{F}_{t, \mathrm{el}}= \begin{cases}k_{t} \dot{\delta_{t}} & \text { if } \dot{F}_{n} \geq 0 \\ k_{t} \dot{\delta_{t}}+F_{t, \mathrm{el}} \frac{\dot{\delta_{n}}}{2 \delta_{n}} & \text { if } \dot{F}_{n}<0 .\end{cases}$

Note that in the unloading phase $\left(\dot{F}_{n}<0\right)$ the tangential force is decremented with respect to the value that would be obtained by the formula corresponding to the loading phase. This arrangement aims to account for the fact that decrements in the contact area reduce the capacity of the contact area to hold tangential deformations by friction. Neglecting this effect leads to undesirable creation of spurious energy (see [41]). At $\delta_{n}=0$, formula 19 is not defined in the unloading phase. Furthermore, the formula cannot be extended with continuity at $\delta_{n}=0$, since in fact the derivative of the force diverges. This can be seen by employing an integrator factor to solve explicitly the linear ordinary differential equation resulting from considering the last unloading phase before losing contact:

$F_{t, \mathrm{el}}=\delta_{n}^{1 / 2} \int a(t) \mathrm{d} t$, 
where the integral denotes any primitive of the integrand $a(t)=8 G^{*} \dot{\delta_{t}}$. This formula shows how $F_{t, \text { el }}$ tends to zero as $\delta_{n}$ tends to zero. On the other hand, deriving the same expression one finds:

$\dot{F}_{t, \mathrm{el}}=\delta_{n}^{-1 / 2} \dot{\delta} \int a(t) \mathrm{d} t+\delta_{n}^{1 / 2} a$

which will diverge for $\delta_{n}$ tending to zero unless $\dot{\delta}$ happens to be an infinitesimal of a higher order than $\delta_{n}$. This function must therefore be used for values of the normal indentation sufficiently far from zero. In practice, however, this is not a problem since the value $\delta_{n}=0$ is never reached in the unloading phase, because it is always the case that the normal force vanishes (and thus the contact is no longer existent) before this value of the indentation is reached for any finite value of the unloading velocity, since otherwise attractive forces would appear in the contact.

The tangential viscous force takes the following form

$F_{t, \mathrm{damp}}=c_{t} \delta_{n}^{1 / 4} \dot{\delta}_{t}$

with

$c_{t}=2 \gamma \sqrt{8 G^{*} M^{*} \sqrt{R^{*}}}$

An expression for $\gamma$ is provided in the following subsection.

\subsection{Viscous damping and restitution coefficient}

A physically consistent choice of the damping coefficient $\gamma$ is required for the system to dissipate energy in a consistent manner. This parameter selection can be guided by describing the corresponding contact energy losses using a restitution coefficient, and establishing a corresponding mapping between restitution and the damping parameter. For the dashpot model as presented here the following empirically derived solution for the expression of $\gamma$ as a function of the normal restitution coefficient, $\varepsilon$ (assumed here to be constant with respect to impact velocity) was proposed in [41]:

$\gamma=\left(\left(1-(1-\varepsilon)^{2} \exp (\alpha)\right)^{-1}-1\right)^{0.5}$,

where $\alpha$ is a tenth-order polynomial in $\varepsilon$ whose coefficients can be found in the the same work. By fixing a coefficient of restitution consistent with available experimental data, it is possible to capture, as a first-order approximation, the energy dissipation of each particular material (see [40] and [18] in the context of fertilizer spreading). Despite existing empirical evidence of its dependence on normal velocity, the assumption that restitution coefficient is constant for a given material is a useful and reasonable alternative, as it is often the case that only a single restitution coefficient is available for empirical characterization of the dissipative properties of a given material combination for the colliding pair.

\subsection{Treatment of contact with rigid surfaces, edges, and kinks}

Typical rotating machinery comprises components like blades, vanes, and rotating disks amongst others. Hence, resolution of contact of individual grains with these components is an important aspect of the proposed model. For evaluation of the contact forces, the contact of a sphere with a rigid plane can be treated as a particular instance of the sphere-sphere contact, in which one of the spheres has infinite radius and stiffness. Therefore, all developments in Sect. 2.1 are directly extendable to particle-surface contacts.

With respect to the detection of contact, the general approach we have followed is based on a Hierarchical Method (see for example [20]) where the control surfaces and components are discretized using a surface mesh or triangulation, and spherical particle contact is checked hierarchically with respective groups of topological entities in the mesh. Specifically, for the model, a common binned data structure has been used with the different types of objects, particles, and triangular elements, in order to efficiently search for potential neighbors. The contact search algorithm is specified a posteriori for the particular type of contact, i.e., particle-face, particle-edge etc., in order to establish pair-wise contacts at each time-step. The result is a robust framework that is able to deal with the four mentioned types of simplical elements: triangles, edges, and vertices, and that can also adequately address the case of multipleelement contributions to the contact with a single particle, and the case of multiple differentiated contacts for a single particle [21]. The data structures, and algorithms have all been implemented through the Kratos Multiphysics software suite [9], and some details with regard to mathematical implementation are presented in Sect. 3.1.

\subsection{Grain motion through ambient fluid}

The motion of a particle embedded in a fluid is typically modeled using a modified form of the Maxey-Riley Eqs. (see [16,28], and [24] for details) obtained by superposing the stresses from the background flow, and the disturbed flow around the particle for a spherical particle. While the full form of the Maxey-Riley equation is relatively complex, requiring specific integration techniques (see for example [43] and [14]), substantial simplifications can be made for the case of a grain moving through ambient air. Thereby, we consider the particle motion to be governed by a simplified equation considering the combined action of drag, Magnus lift induced due to particle rotations, and gravity as follows: 
$m_{p} \frac{d \mathbf{v}_{p}}{\mathrm{~d} t}=\mathbf{F}_{\mathrm{drag}}+\mathbf{F}_{\text {magnus }}+m_{p} \mathbf{g}$,

$\mathbf{F}_{\mathrm{drag}}=-\frac{1}{2} \rho_{f} C_{D}\left(\frac{\pi D_{p}^{2}}{4}\right)\left\|\mathbf{v}_{p}\right\| \mathbf{v}_{p}$,

$\mathbf{F}_{\text {magnus }}=\frac{1}{2} \rho_{f} C_{L R}\left(\frac{\pi D_{p}^{2}}{4}\right)\left\|\mathbf{v}_{p}\right\| \frac{\omega_{p} \times \mathbf{v}_{p}}{\left\|\omega_{p}\right\|}$,

where $C_{L R}$ denotes a lift coefficient dependent on the rotation of the particle. It can be expressed in form of an empirically derived correlation proposed by [34], given by the following expression:

$C_{L R}=0.45+(2 \Omega-0.45) \exp \left[-0.075 \Omega^{0.04} \operatorname{Re}_{p}^{0.7}\right]$

The term $\Omega$ is a non-dimensional particle spin parameter which can be used to quantify the extent of lift the particle will experience due to spin, and is defined as follows:

$\Omega=\frac{D_{p}\left\|\omega_{p}\right\|}{2\left\|\mathbf{v}_{p}\right\|}$,

and $\operatorname{Re}_{p}$ denotes the particle slip velocity based Reynolds number, as follows:

$\operatorname{Re}_{p}=\frac{\rho_{f} D_{p}\left\|\mathbf{v}_{\mathbf{p}}\right\|}{\mu_{f}}$.

It can be seen, from Eq. 29, that the lift coefficient due to particle spin has a nearly linear variation with the spin parameter $\Omega$.

The drag coefficient is estimated using a correlation based on the slip-Reynolds number $\mathrm{Re}_{p}$, as proposed by [17], as follows:

$C_{D}=\frac{24}{\operatorname{Re}_{p}}\left(1+0.1806 \operatorname{Re}_{p}^{0.6459}\right)+\frac{0.4251}{1+\frac{6880.95}{\operatorname{Re}_{p}}}$.

We note that while alternative correlations may exist for $C_{L R}$ and $C_{D}$, the specific choices as in Eqs. 29 and 32 were made owing to their applicability over a relatively wide range of Reynolds numbers.

The particle response time to the background flow for such analysis is denoted as $\tau_{p}$ such that $\tau_{p}=\rho_{p} D_{p}^{2} / 18 \mu_{f}$ (see [8]). Using this definition for $\tau_{p}$, and the definition of $\operatorname{Re}_{p}$ as in Eq. 31, and assuming a characteristic length scale $L$ and characteristic velocity scale $U$ (thereby defining a characteristic time-scale $T=L / U$ ), we get a non-dimensionalized form of the motion equation for the particle as follows:

$$
\begin{aligned}
\frac{d \mathbf{v}_{p}^{*}}{d t^{*}}= & -\frac{C_{D} \operatorname{Re}_{p}}{24}\left(\frac{T}{\tau_{p}}\right) \mathbf{v}_{p}^{*} \\
& +\frac{C_{L R} \operatorname{Re}_{p}}{24}\left(\frac{T}{\tau_{p}}\right) \frac{\omega_{p}^{*} \times \mathbf{v}_{p}}{\left\|\omega_{p}^{*}\right\|}+\frac{\mathbf{g} L}{U^{2}}
\end{aligned}
$$

Note that the term $T / \tau_{p}$ is the particle Stokes number, and additionally, while the $\operatorname{drag} C_{D}$ and Magnus lift coefficients $C_{L R}$ appear in the non-dimensional parameters, the underlying independent variables controlling their magnitudes are $\operatorname{Re}_{p}$, and $\Omega$. Hence we find that the particle trajectory is characterized by four non-dimensional groups- $-\mathrm{Re}_{p}, S t_{p}$, $\Omega$, and $g L / U^{2}$-and their action on the particle trajectories are through the lumped non-dimensional parameters:

$$
\begin{aligned}
-\beta_{d} & =\frac{C_{D}\left(\operatorname{Re}_{p}\right) \operatorname{Re}_{p}}{24 S t_{p}} \\
-\beta_{m} & =\frac{C_{L R}\left(\Omega, \operatorname{Re}_{p}\right) \operatorname{Re}_{p}}{24 S t_{p}} \\
-\beta_{g} & =\frac{g L}{U^{2}} .
\end{aligned}
$$

Here, $\beta_{d}$ indicates the dominance of drag forces over particle inertia, $\beta_{m}$ indicates the dominance of Magnus lift forces over inertia, and $\beta_{g}$ indicates the dominance of gravity over particle inertia.

The rotational motion of the particle in a flow field has not been investigated in much detail, and only a few simple expressions are available to set-up a reasonable approximation to the rotational motion equation of the spherical particle (see $[11,15]$ for details). We employ here the following form:

$I_{p} \frac{d \omega_{p}}{\mathrm{~d} t}=-\alpha \mu_{f} D_{p}^{3} \omega_{p}$

$\alpha=2.01\left(1+0.201 \sqrt{\operatorname{Re}_{\text {spin }}}\right)$,

where a rotational Reynolds number $\mathrm{Re}_{\text {spin }}$ can be defined as follows:

$\operatorname{Re}_{\text {spin }}=\frac{\rho_{f}\left\|\omega_{p}\right\| D_{p}^{2}}{4 \mu_{f}}$

As a further remark, assuming that the sphere rotation is resolved about the principal axis of rotation, then Eq. 34 can be directly solved as a first-order ordinary differential equation, and the solution to the angular velocity will be of the form $\omega_{p}(t)=\omega_{0} \mathrm{e}^{-\eta t}$. The time constant of decay of the angular velocity under the action of the viscous torque on the sphere can then be directly quantified as follows:

$T_{\omega}=\frac{1}{\eta}=\frac{I_{p}}{\alpha \mu_{f} D_{p}^{3}}$. 


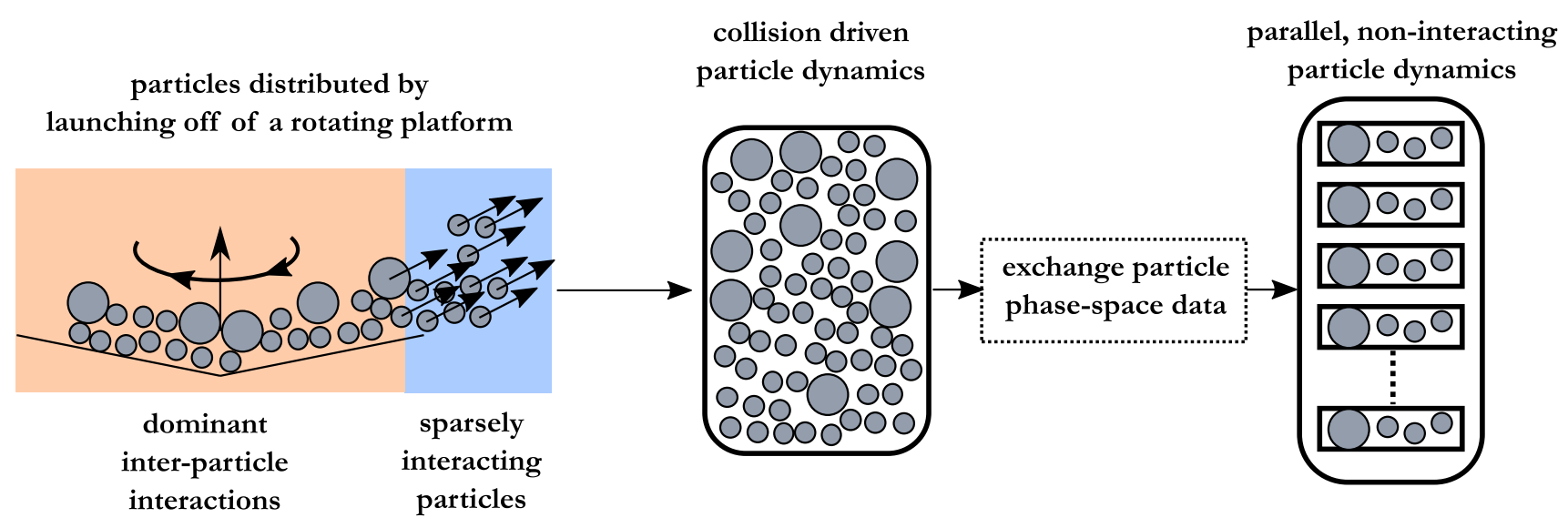

Fig. 1 A schematic representation of the partitioned computation scheme, with the contact-dominant dynamics and the in-flight dynamics being dealt with as distinct modules, with exchange of particle phsae-space data between the two

\section{Simulation framework}

\subsection{A modular simulation algorithm}

The overall simulation framework can be formulated by taking advantage of the two distinct regimes of particle dynamics as motivated in Sect. 1. The central idea is illustrated in form of a schematic in Fig. 1. The dynamics of the particles on the disk comprises particle-particle, and particle-surface contact interactions. For a collection of $N_{p}$ particles, with the i'th particle denoted as $\mathcal{P}_{i}$, the overall motion equation can be written as follows:

$$
\begin{aligned}
m_{i} \frac{d \mathbf{v}_{i}}{\mathrm{~d} t}= & \sum_{j=1}^{N_{s}} \overbrace{\mathcal{I}\left(\mathcal{P}_{i}, \mathcal{S}_{j}\right)}^{\text {check contact }} \underbrace{\left[\mathbf{F}_{i j s, \text { contact }}\right]}_{\text {particle-surface }} \\
& +\sum_{j \neq i, j=1}^{N_{p}} \overbrace{\mathcal{I}\left(\mathcal{P}_{i}, \mathcal{P}_{j}\right)}^{\text {check contact }} \underbrace{\left[\mathbf{F}_{i j, \text { contact }}\right]}_{\text {particle-particle }}
\end{aligned}
$$

$\frac{d \mathbf{x}_{i}}{\mathrm{~d} t}=\mathbf{v}_{i}$

$$
\begin{aligned}
I_{p} \frac{d \boldsymbol{\omega}}{\mathrm{d} t}= & \sum_{j=1}^{N_{s}} \overbrace{\mathcal{I}\left(\mathcal{P}_{i}, \mathcal{S}_{j}\right)}^{\text {check contact }} \underbrace{\left[\mathbf{M}_{i j s, \text { contact }}\right]}_{\text {particle-surface }} \\
& +\sum_{j \neq i, j=1}^{N_{p}} \overbrace{\mathcal{I}\left(\mathcal{P}_{i}, \mathcal{P}_{j}\right)}^{\text {check contact }} \underbrace{\left[\mathbf{M}_{i j, \text { contact }}\right]}_{\text {particle-particle }}+\mathbf{M}_{i, \text { external }},
\end{aligned}
$$

where $\mathcal{S}_{j}$ denotes the $j^{\prime}$ th control surface of the machin-

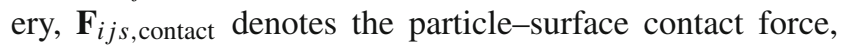
$\mathbf{F}_{i j \text {,contact }}$ denotes the particle-particle contact force (as described in Sects. 2.1 and 2.2), and $\mathbf{M}_{i j s \text {, contact }}, \mathbf{M}_{i j \text {, contact }}$ are the corresponding contact moments (as in Eq. 15). The function $\mathcal{I}(\cdot, \cdot)$ is generalized contact indicator function. Specifically, $\mathcal{I}\left(\mathcal{P}_{i}, \mathcal{S}_{j}\right)$ is equal to unity when the i'th particle is in contact with the $j^{\prime}$ th surface, and zero otherwise. Similarly, $\mathcal{I}\left(\mathcal{P}_{i}, \mathcal{P}_{j}\right)$ is equal to unity when i'th and j'th particles are in contact, and zero otherwise. Furthermore, any control surface of the machinery appears in the simulations in its discretized form (unless if it is a planar surface), and generally each surface $\mathcal{S}_{j}$ is therefore to be thought of as a collection of triangular surface elements $\mathcal{S}_{j}=\cup_{i=1}^{N_{s}} \mathcal{T}_{w, j}$, as discussed in Sect. 2.3. The contact indicator function evaluation is then a function of the spherical particle contacts with these triangular surface elements.

The dynamics of the grains on the rotating disk is then numerically integrated within the Kratos Multiphysics software suite [9], using an explicit Euler integration scheme, such that

$$
\begin{aligned}
{\left[\mathbf{v}_{i}^{N+1}-\mathbf{v}_{i}^{N}\right]_{\text {on-disk }}=\frac{\Delta t_{c}}{m_{i}} \mathbf{F}_{i}^{N} } \\
\mathbf{F}_{i}^{N}=\sum_{j=1}^{N_{s}} \mathcal{I}\left(\mathcal{P}_{i}^{N}, \mathcal{S}_{j}^{N}\right)\left[\mathbf{F}_{i j s, \text { contact }}\right]^{N} \\
\quad+\sum_{j \neq i, j=1}^{N_{p}} \mathcal{I}\left(\mathcal{P}_{i}^{N}, \mathcal{P}_{j}^{N}\right)\left[\mathbf{F}_{i j, \text { contact }}\right]^{N}
\end{aligned}
$$

where the superscripts $N$ on the particle and surface variables $\mathcal{P}$ and $\mathcal{S}$, respectively, denote that the current particle and surface positions and velocities are directly used for evaluating contact checks and estimating contact forces. The parameter $\Delta t_{c}$ is the numerical integration time-step for the on-disk, contact-driven, particle dynamics integration. The rotation motion described in Eq. 40 is integrated in the same manner, and hence has been not explicitly written out here.

For guiding the choice of $\Delta t_{c}$, it is required to have an estimate of the characteristic contact duration. Denoting this 
duration as $\delta t_{c}$, it is typically assumed that $\Delta t_{c}$ should resolve the contact duration well and $\Delta t_{c}=\delta t_{c} / N_{c}$. For our simulations $N_{c} \approx 10-20$. The contact duration, can be estimated as a function of incident normal velocity and material properties, using the mechanics of compression and recovery during contact (see [2, 27], and [22]). For the Hertzian contact mechanics, this can be estimated as follows:

$\left.\delta t_{c}\right|_{\text {elastic }}=2.586\left(\frac{M^{*}}{E^{*} R^{*}}\right)^{2 / 5} u_{n 0}^{-1 / 5}$,

where $u_{n 0}$ is the incident relative normal velocity.

As the particle velocities and angular velocities evolve on the disk through contact interactions, the particles reach the edge of the rotating disk. At the instant of particle launchoff of the disk, the particle phase-space data is recorded and the particle is removed from the 'on-disk' calculations. The recorded phase-space information is then employed to calculate the trajectory of the particle under the action of fluid forces as modeled in Sect. 2.4. An explicit fourth-order Runge-Kutta integration scheme is used to obtain the trajectory of the particles until they reach the target region. The generalized form of the explicit integration scheme is as follows:

$$
\begin{aligned}
& {\left[\mathbf{v}_{i}^{N+1}-\mathbf{v}_{i}^{N}\right]_{\text {in-flight }}=\frac{\Delta t_{f}}{6 m_{i}}\left[\mathbf{k}_{1}+2 \mathbf{k}_{2}+2 \mathbf{k}_{3}+\mathbf{k}_{4}\right]} \\
& \mathbf{k}_{1}=\mathbf{F}_{i}\left(t_{n} ; \mathbf{v}_{n}\right) \\
& \mathbf{k}_{2}=\mathbf{F}_{i}\left(t_{n}+\frac{\Delta t_{f}}{2} ; \quad \mathbf{v}_{n}+\frac{\Delta t_{f}}{2} \mathbf{k}_{1}\right) \\
& \mathbf{k}_{3}=\mathbf{F}_{i}\left(t_{n}+\frac{\Delta t_{f}}{2} ; \quad \mathbf{v}_{n}+\frac{\Delta t_{f}}{2} \mathbf{k}_{2}\right) \\
& \mathbf{k}_{4}=\mathbf{F}_{i}\left(t_{n}+\Delta t_{f} ; \quad \mathbf{v}_{n}+\Delta t_{f} \mathbf{k}_{3}\right)
\end{aligned}
$$

where $\mathbf{F}_{i}$ is the sum of the fluid forces acting on the particle as presented in Eq. 26, and $\Delta t_{f}$ is the integration time-step for the flight dynamics of the particles. For brevity, the position and the rotational momentum integration equations, as well as the explicit dependence of forces and moments on position have not been presented here, but it is a direct extension of the scheme presented here, and the models described in Sect. 2.4. A central aspect of the simulation framework is such that $\Delta t_{c}$ and $\Delta t_{f}$ are not equal, and the selection of these time-steps take advantage of the fact that there are two distinct timescale and dynamics regimes within the overall simulation.

Specifically, for the simulation of the 'in-flight' dynamics of the grains, the framework takes into account the sparsity of inter-particle interactions. This is utilized to process the grains as non-interacting particles, in a massively parallel manner. The ensemble of particles that are launched, is divided into a set of $N_{\text {proc }}$ processes, and all of these sets are computed simultaneously (see Fig. 1). For estimation of the final distribution of particles to the target surface, these particles can be divided in any manner. However, if the time of launch is also to be incorporated in the simulations, then a possible approach is to divide particles into processors such that all particles in a process have been released within the same time-window. This makes the computations modularwith the on-disk and in-flight dynamics treated as different modules. Thus the overall computations from grain release onto the rotating machinery, to the spread of grains on a target surface, becomes efficient and scalable to a large number of grains. While simple, embarrassingly parallel strategies actually work very well for this, we observed that as the particle numbers get larger, the balancing of the computational load amongst the various parallel processes may be increasingly critical since all particles may reach the target at different instants in time.

An additional factor which is crucial for numerical efficiency to the modular framework presented here is the selection of an integration time-step for the individual grain dynamics. For the 'on-disk' dynamics of the particles, the individual particle-particle, and particle-surface collisional interactions place a restriction on the upper-bound of the time-step $[19,35]$. Thus to ensure sufficient resolution of the collisions using the force-deformation type contact model, the time-steps are required to be smaller than the characteristic contact durations between particles and surfaces $[25,38,39]$. This is no longer necessary, however, for the inflight dynamics, since no contact interactions are presumed. This allows for bigger time-steps than the contact-limited time-steps, for the flight dynamics.

As mentioned earlier, typically we assume that $\Delta t_{c}=$ $\delta t_{c} / N_{c}$ where $N_{c}$ is the number of time-steps required to resolve the contact, and integrate the forces accurately. On the other hand, for sparsely interacting systems, with contact events neglected, the time-step $\Delta t_{f}$ can be chosen to be $N_{f c} \Delta t_{c}$, since the requirement of resolving contact duration is relaxed. Furthermore, for an arbitrarily chosen particle in the system, and for an overall simulation duration $T_{0}$, we can assume on an average that $T_{0}=T_{c}+T_{f}$. Here $T_{c}$ is the duration for which the particle is contact driven, and $T_{f}$ is the duration where in the particle is in-flight. Based on these, and based on the modular computation framework, an essential feature of this framework can be outlined, by defining the baseline proportion of computation steps for the single particle as follows:

$$
\begin{aligned}
C_{r} & =\frac{\frac{T_{c}+T_{f}}{\Delta t_{c}}-\frac{T_{c}}{\Delta t_{c}}-\frac{T_{f}}{\Delta t_{f}}}{\frac{T_{c}+T_{f}}{\Delta t_{c}}} \\
& =\frac{1-\frac{1}{N_{f c}}}{1+\frac{T_{c}}{T_{f}}}
\end{aligned}
$$


While this simple analysis does not include aspects like parallel processing, and data-exchange overhead, it points out a couple of essential aspects of the framework. Firstly, if $N_{f c} \approx 1$ the efficiency approaches 0 , while for high $N_{f c}$ values the efficiency will increase. Therefore, attaining larger integration time-step ratios is desirable. At the same time, if $T_{f}$ is much smaller than $T_{c}$, the efficiency will go down. Therefore, it is also desirable to employ such a framework for processes that have a substantial extent of non-contactdriven dynamics.

\subsection{Initial conditions}

The particles are initialized using an auxiliary planar mesh, previously generated by triangulating a designated input area. The particles are initially placed at the mesh nodes and moved away from them at a controlled velocity until a certain activation condition is attained. Once this condition is attained, the particles are released, their initial conditions are set, contact interaction is activated and a new particle is seeded at the same mesh node. In all the validation examples, the particle configuration at the instant of activation had no overlaps between any pair of neighboring particles, so as to avoid any numerical instabilities owing to initial (artificial) contact deformations. The input surface was chosen to be circular, so as to evenly drop particles into a conical funnel. The funnel's opening diameter was provided in the corresponding sources, though the inclination of its walls was estimated based on pictures.

Every particle is initialized having a diameter that is sampled from a known probability distribution. A lognormal distribution was chosen for the validation examples, making its mean and standard deviation match those provided in their corresponding sources. The particle feed rate was imposed in an average sense. That is, the number of particles injected per second (which can be exactly imposed, as long as the release condition is met quickly enough) was fixed to such a value that the average mass per second matched the desired mass feed rate. Since this number is defined using the properties of the sampled diameter distribution (which has simple formulas in the lognormal case), this calculation was easily done.

In reality these particles, as mentioned earlier, are injected onto the machinery by some hopper or conveyor which has not been fully modeled here. Alternative release strategies based on modeling the gravity-driven discharge from a filled up containing cylinder have been explored by [6] and by [45]. No attempt has been made here to model the discharge process itself, since the primary focus of the framework was to capture the fate of the grains after they had been launched onto the distributing machinery.

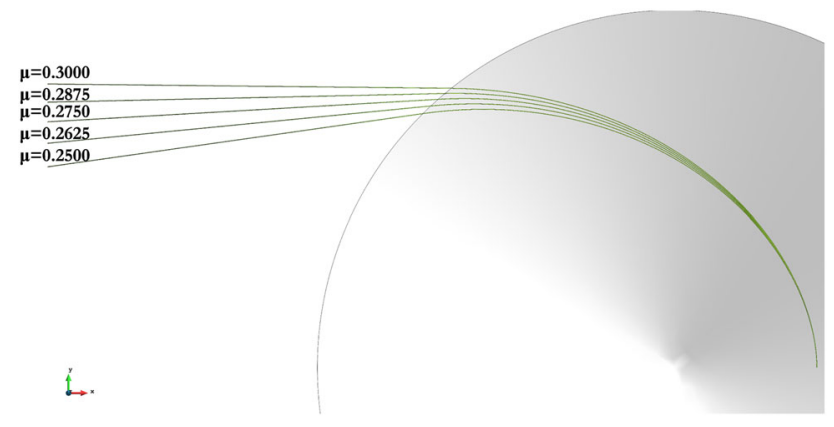

Fig. 2 Particle trajectories obtained from the semi-analytical equations for particles on a rotating cone, calculated for varying friction coefficients

\subsection{Mesh refinement and on-disk dynamics}

The control surfaces interacting with the particles are not necessarily flat, and since a geometrically discretized form of these surfaces are employed in the simulations, it is critical to illustrate the effect of the discretization error associated on the overall particle dynamics, and their emergent properties. This is an aspect that has not received sufficient attention in the existing literature, and hence a numerical comparison study to explore this aspect in detail has been established here.

For this purpose, an analytical model for the motion of a single particle moving along a rotating, conical surface, has been outlined. The details of the formulation, and the expressions, have been presented in Appendix 1. Following which, a geometrically discretized form of the same conical surface, was used within the proposed DEM framework, and the 'on-disk' dynamics of particles was simulated, and their trajectories compared with the analytical solution. Figure 2 shows the superposition of five different numerical solutions of the system for varying values of the coefficient of friction. In a clockwise sense, the successive trajectories correspond to the following increasing succession of values of the coefficient of friction: $0.25,0.2625,0.275$, $0.2875,0.3$. The following list summarizes the main features of the numerical set-up for the corresponding DEM calculations, that was devised in order to achieve a fair comparison and better isolate the mentioned effect of the mesh discretization.

- A one-node inlet mesh was used to generate the particles. Upon injection, they were allowed to drop under gravity until they hit the surface. At this point, the IR initial velocity was set to be the same as the one used for the analytical trajectory calculations.

- The particle rotation degrees of freedom were eliminated in order to obtain a sliding behavior similar to that of the point-mass of the analytical solution. 


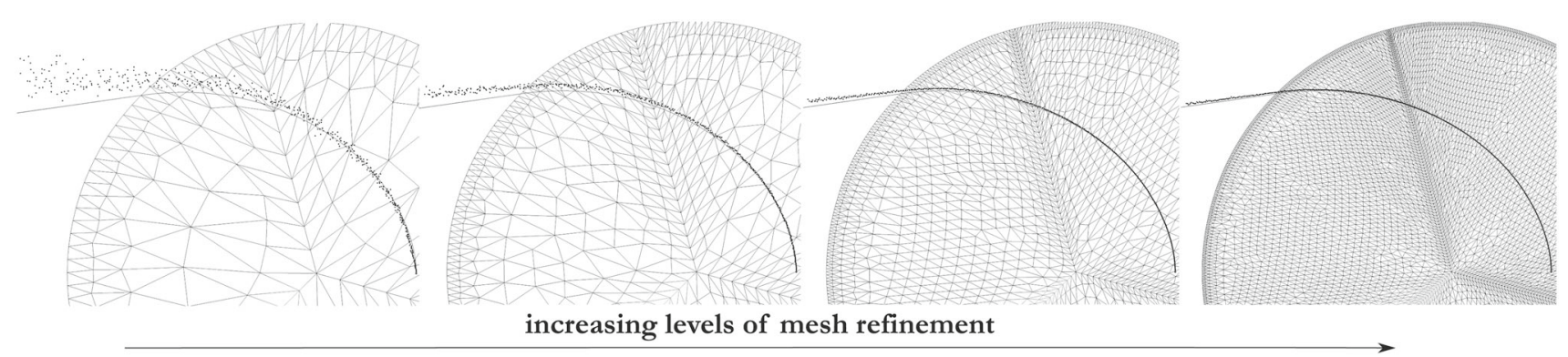

Fig. 3 Particle trajectories, compared with the corresponding trajectory obtained from the semi-analytical approach, compared for increasing levels of refinement of the conical surface mesh (normalized maximum element size $\xi$ reduces from left to right)

- Multiple non-interacting particles, instead of just one particle, were used so as to get well-averaged ensemble statistics.

For the comparisons, the particle radius, non-dimensionalized by the maximum radius of the cone, denoted by $\delta$ was set at 0.005 . The restitution coefficient $\varepsilon$ was set at 0.2 , and used to estimate the damping coefficient as described in Sect. 2.2. For the multiple refined meshes, the friction coefficient was set at 0.25 . The linearized natural frequency of the elastic force component, normalized by the angular velocity, was set at $2.0 \times 10^{-6}$. The magnitudes of these parameters were chosen to fall within the typical ranges encountered in fertilizer spreading applications. For instance, the values of $\gamma$ and $\varepsilon$ are taken from average values mentioned in [18], and the value of $\Omega$, which is defined as (see, e.g., [37])

$\Omega=\left(\frac{3 k_{n}}{2 M}\right)^{1 / 2}\left(\delta_{S}\right)^{1 / 4} \omega$

is made to coincide with the value used in [44]. Finally, the level of refinement is characterized by the normalized maximum element size, denoted by $\xi$ and is non-dimensionalized with respect to the maximum radius of the cone. This is the only parameter that is varied in the present analysis.

The corresponding numerical simulation results for four levels of refinement of the conical surface mesh have been presented in Fig. 3. It is seen that coarser meshes lead to an added randomness in the particle motion, owing to a more pronounced sequence of collisions originating from sharper changes in the element normals of the triangular elements discretizing the surface. A comparison of the average trajectory of the particles, with the trajectories presented in Fig. 2, indicates a phenomenological effect of an apparent enhancement of friction, which causes particle trajectories for coarser meshes to deviate from the true solution. The corresponding average particle velocity magnitude and direction for the different meshes, is compared with that obtained from the semi-analytical approach in Fig. 4. Herein again, a reduc- tion in velocity with coarsening of the mesh, supports the aforementioned phenomenology of an augmented friction.

\section{Numerical simulations and validation}

\subsection{Representative dynamics of grains}

Representative numerical simulations were performed with realistic system parameters derived from the studies reported by $[46,47]$ and [3], to demonstrate and validate the proposed framework. These examples, as mentioned before, pertain to fertilizer spreading systems, which have been sufficiently well documented in the literature, with sufficient extent of experimental data. We use the modular simulation framework to compute the distribution of fertilizer grains dropped onto a conical, rotating disk, with multiple radial vanes. The simulation parameters for the two cases described here have been compiled in Table 1. Successive snapshots of the grain dynamics on the disk, with inter-particle and particle-vane collision interactions, and enduring contact with the disk surface, have been presented in Fig. 5. This figure corresponds to the model described in Case 1 in Table 1. Each panel in Fig. 5 represents approximately 20,000 particles. The corresponding spread pattern on the ground has been shown for Case 1 , in Fig. 6, on the right panel. To understand how the incorporation of Magnus forces affect the final distribution, a set of control simulations were run where the Magnus lift force was set to zero. The corresponding spread pattern has also been presented in Fig. 6 on the left panel. It can be seen that the inclusion of the lift forces causes the particles to spread out, leading to a wider scatter in the on-ground distribution pattern. We remark here that the contact interactions, and the complexity of the overall contact-driven dynamics of the grains, do lead to significant levels of rotational velocities for the particles at the instant of launch-off the disk. Therefore the incorporation of the Magnus effect is a very relevant factor. Furthermore, any inaccuracies in predicting the particle velocities and rotational velocities at the instant of launch, will manifest themselves noticeably due to the action of the 
Fig. 4 Variation in velocity magnitude and velocity direction, with mesh refinement. The top two panels denote the velocity magnitude at the edge of the disk, and the difference of the calculated magnitude with the semi-analytically obtained value. The bottom panels show the corresponding values for velocity direction (angle with horizontal)
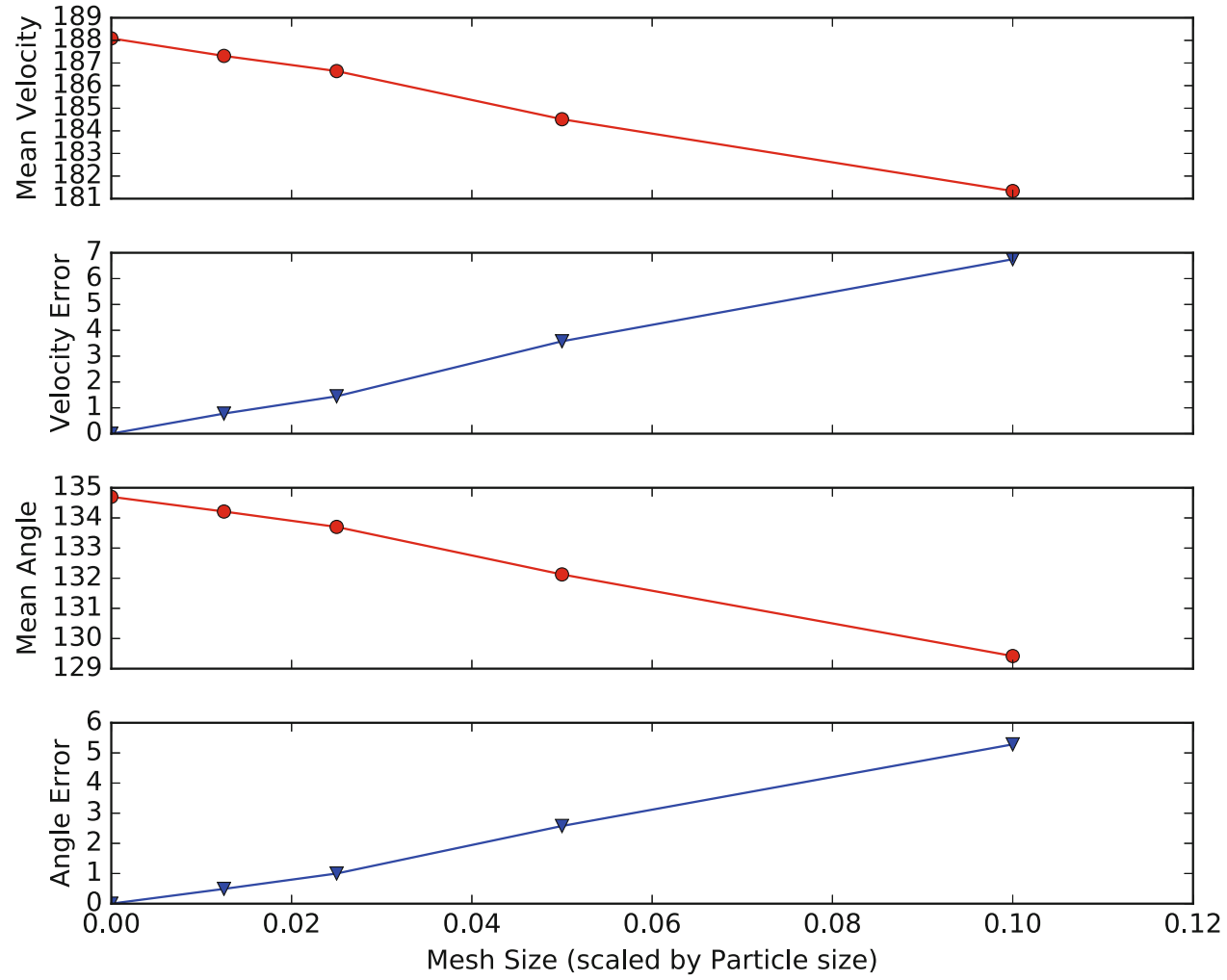

lift forces. In order to further illustrate the effect of the Magnus lift, in-flight pathlines of particles from simulations corresponding to Case 2 , have been presented for cases with and

Table 1 A description of primary simulation parameters extracted from the experimental studies documented in [46], and [3], which have been used for constructing the numerical experiments herein

\begin{tabular}{lll}
\hline Parameter & Case 1 [46] & Case 2 [3] \\
\hline Spreader disk geometry & & \\
Disk radius (m) & 0.29 & 0.1524 \\
Height above ground (m) & 0.28 & 1.0 \\
Number of vanes & 2 & 4 \\
Height of vanes (m) & 0.036 & 0.025 \\
Angle of cone (rad) & 0.157 & 0.0 \\
Inlet parameters & & \\
Radius of inlet plane (m) & 0.0145 & 0.0065 \\
Offset from disk center (m) & 0.1 & 0.05 \\
$\quad$ Mass feed rate & $0.11 \mathrm{~kg} / \mathrm{s}$ & \\
Dynamics of disk & & 540,810 \\
Disk rotation (rpm) & 500 & $0.4-0.6$ \\
Dynamic friction & 0.35 & \\
Fluid properties & & \\
Air viscosity & $0.00001 \mathrm{~m}^{2} / \mathrm{s}$ & \\
Air density & $1.225 \mathrm{~kg} /{ }^{3}$ & \\
Ambient air flow & Stagnant & \\
\hline
\end{tabular}

without the Magnus lift force in Fig. 7. It is evident that the resultant effect is that the particle flight duration before landing on the target surface is increased markedly, and the inflight dynamics of the particles becomes more tortuous, with higher loops, than in the case where only drag is dominant.

An interesting aspect for such simulations is the convergence of the emergent spread distribution characteristics. The aspect of convergence in these simulations involves two primary factors, and for the purpose of these applications, convergence is checked for in terms of the distribution statistics of the particle spread. Firstly, for a given set of simulation parameters, the statistics evolve with increase in the number of particles simulated, until convergence is reached. Secondly, and as discussed in Sect. 3.3, the on-disk dynamics will vary with mesh refinement, and thus the convergent behavior of distribution statistics with respect to refinement of the rotating machinery surface mesh is of interest. While the aspect of mesh refinement has been discussed in greater detail in Sect. 3.3, we present here representative trends for both of these factors in terms of distribution statistics. In order to numerically characterize the distribution, we employ the following two derived parameters previously proposed in [45,46]:

$\begin{aligned} \bar{R} & =\sum_{i} \sum_{i} \bar{m}_{i j} r_{i j} \quad \text { Average radius } \\ \xi & =\sum_{i} \sum_{j} \bar{m}_{i j}\left|r_{i j}-\bar{r}\right| \quad \text { Radial width },\end{aligned}$ 


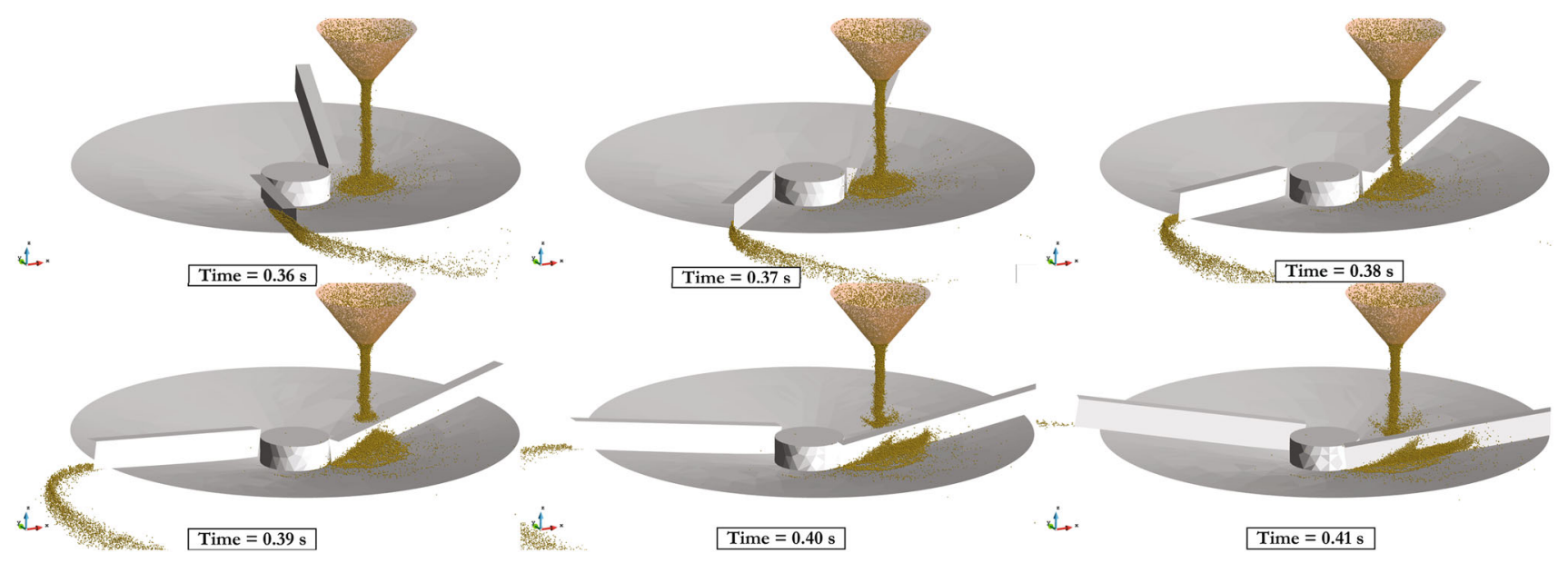

Fig. 5 Snapshots of the on-disk dynamics of the grains for 5 successive time instances for the two-vaned disk as presented in [46]
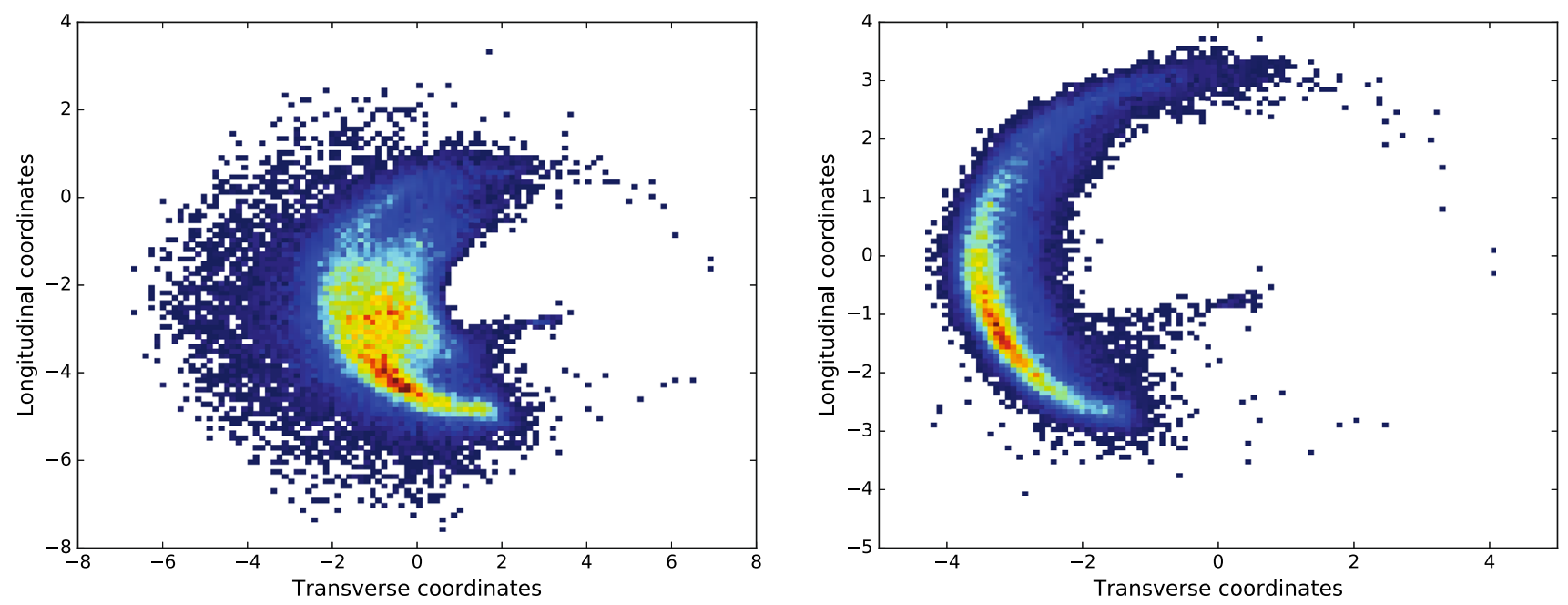

Fig. 6 The spread pattern on-ground for simulations with Magnus lift forces taken into account (left), and without Magnus lift forces (right), for the two-vaned rotary spreader with angular velocity of $300 \mathrm{rpm}$

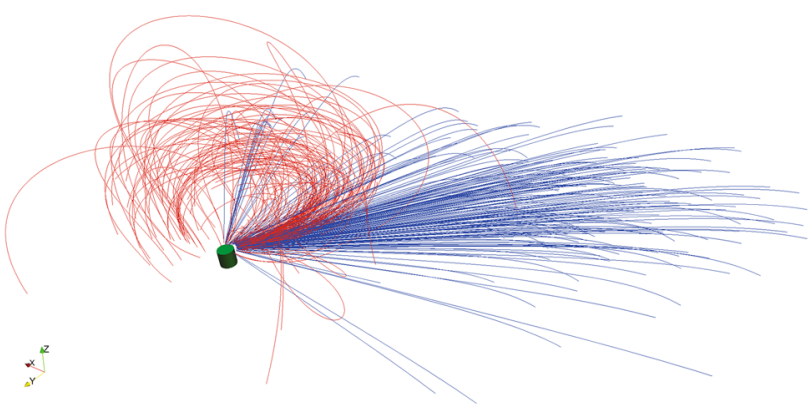

Fig. 7 A representative sample of particle pathlines for simulations with and without the consideration of Magnus lift forces (red and blue, respectively). The disk is indicated in green. (Color figure online)

where $\bar{m}_{i j}$ is the mass fraction contained in the $i, j$-th interval in space, and $r_{i j}$ is the radius of the $i, j$-th interval. The relative variations of both of these parameters, with respect to increasing number of particles from $\approx 11,000$ to $\approx 250,000$, and with respect to mesh size parameter ranging from 0.002 to 0.02 have been compiled in Fig. 8. It is observed that mesh refinement has a more prominent effect on the chosen distribution parameters, as compared to increasing number of particles. Coarser meshes end up artificially enhancing the friction, and causing random variations in particle trajectories as explained in detail in Sect. 3.3. This leads to slower-but more randomly varying — velocities, hence the radius reduces, but width increases with coarsening. While the exact scatter of the particles do end up varying as the number of particles increase, it is observed that the averaged distribution properties generally tend to be less sensitive to variations in the number of particles. Understandably, however, remarkably low numbers of particles will lead to substantial variations in these averaged properties as welland hence all cases have been ensured to be away from that regime. 

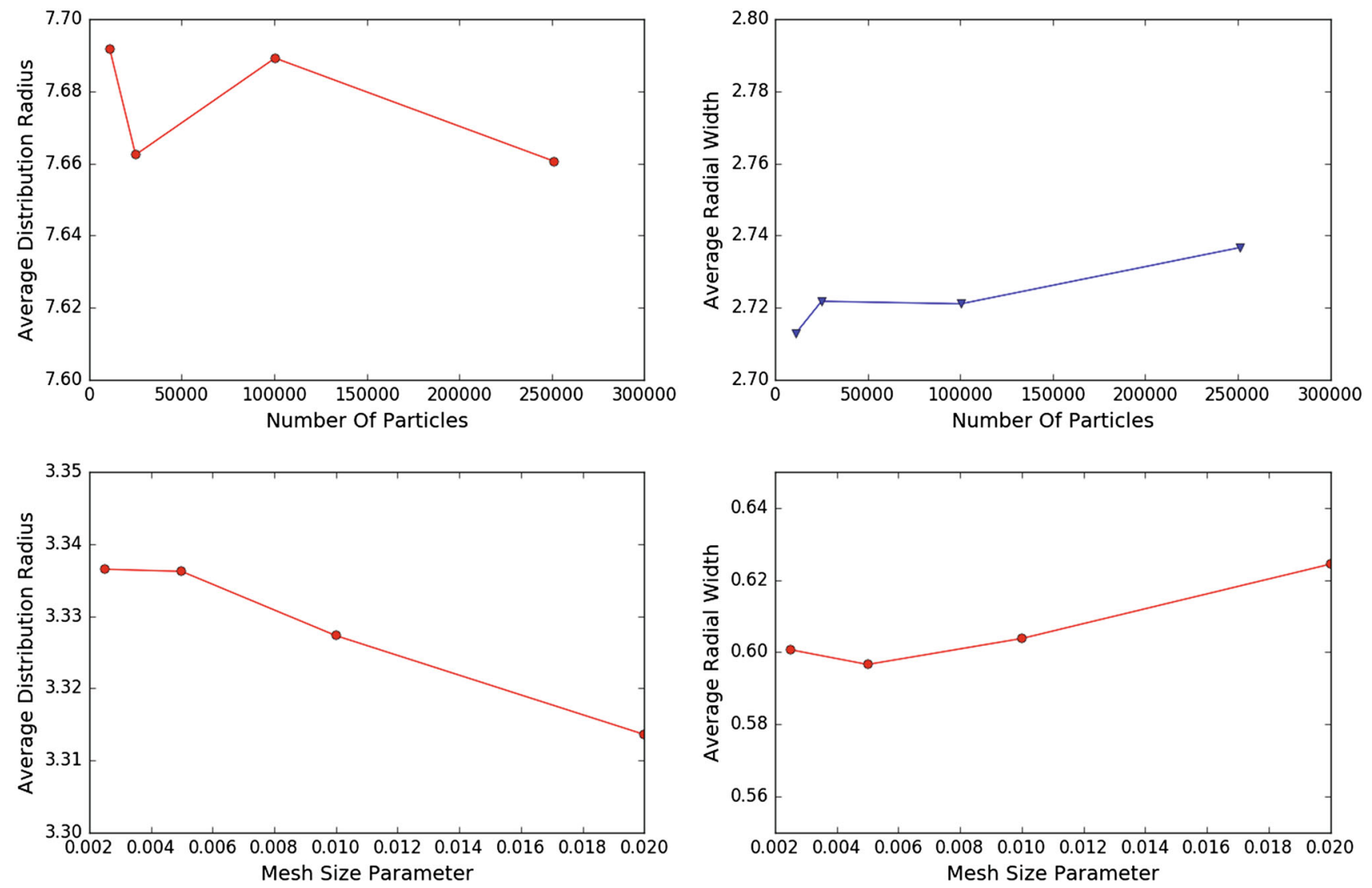

Fig. 8 Variation of average radial distribution and radial width defined in Eqs. 51 and 52, respectively, with number of particles (top, left, and right), and with mesh refinement of the disk surface (bottom, left, and right)

\subsection{Contact dominated, on-disk dynamics}

In the study presented in [46] and [45], an arrangement of cylindrical collectors were employed surrounding the rotating disk to capture the particles at the instant of launch-off from the disk. An alternative set of collecting bins were placed on the ground to capture particles at the instant of landing. The reported experimental distribution patterns of the grains captured by the cylindrical collector arrangement indicate a characteristic triangular (or bell-type) shape. In order to benchmark the predicted particle fate at the end of the ondisk calculations, a corresponding numerical experiment was performed to simulate the particle capture by collectors at the edge of the disk. In this simulation, the particle positions were distributed across circumferential, discretized bins, and the resulting statistics were compared directly with the experimental distribution data presented in the original study [46]. The results for three different disk angular velocities - 300, 500, and $650 \mathrm{rpm}$ - have been presented in Fig. 9. The results show extremely good agreement with experiments, and the small differences observed are possibly due to the effect of the surface discretization, and the numerical inaccuracies emanating from the particular treatment of the contact forces.

\subsection{In-flight dynamics}

Contrary to the on-disk dynamics, a corresponding quantitative validation of the in-flight dynamics, and the resultant distribution patterns is more complicated. The exact treatment of the fluid forces on the particle differ notably between the available studies in the literature, with a commonly favored approach being that of a common resistance coefficient to model the drag on the particle [3,6]. Additionally, the dynamical nature of the particle trajectory equations as presented in Eq. 26 oftentimes leads to small errors in calculated particle initial velocities and forces manifest themselves notably in the trajectory of the particles. Hence, a qualitative comparison was intended for this part of the framework, and the data presented in the study by [3] was chosen for the purpose of comparison. In this study, both the on-disk as well as the in-flight dynamics of a wide range of particles spread using a four-vane rotary spreader were characterized. The subset of materials chosen for our comparison has been outlined in Table 2 . The study characterized extensively the radial distribution of the particles on ground, and reported data not only from experiments, but also from simple numerical analysis. Their analysis included particle dynamics on 


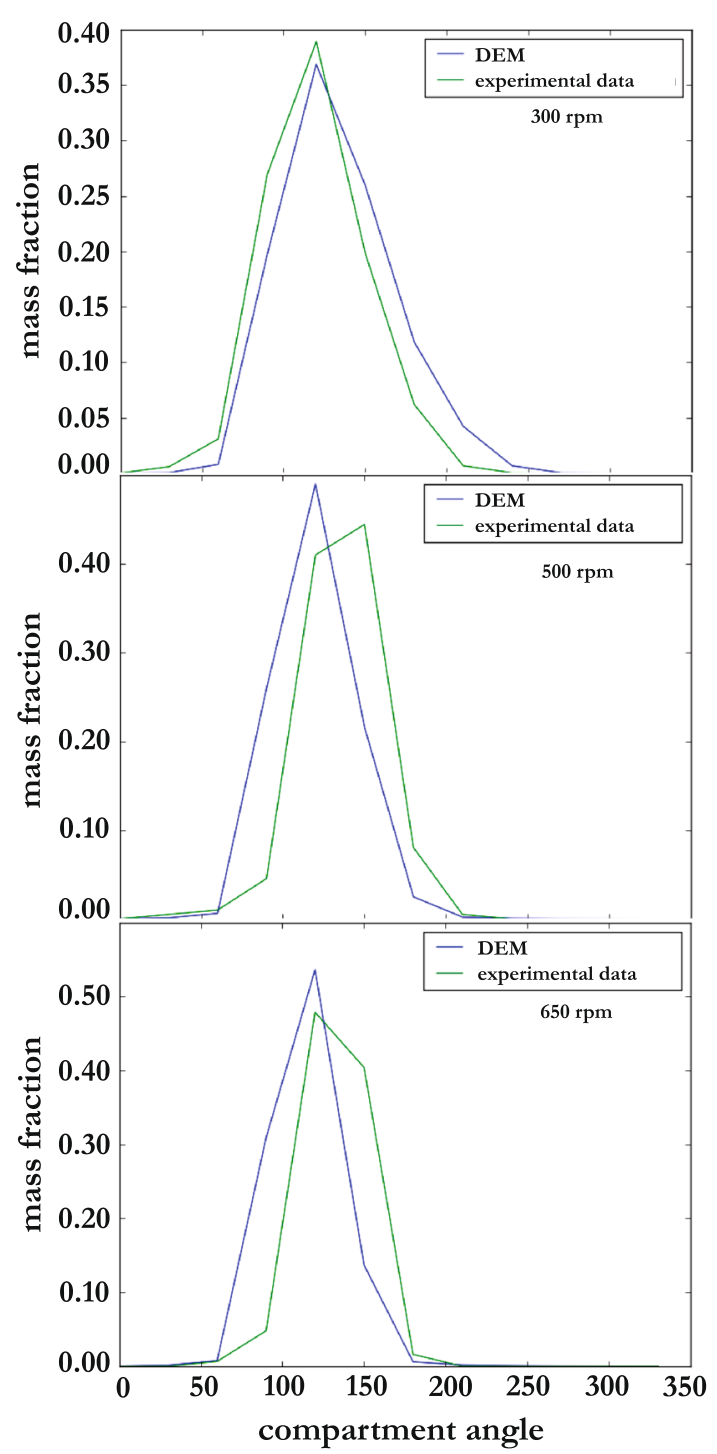

Fig. 9 Validation of angular particle mass-fraction distribution immediately post launch-off from the spreader, with experimental data reported in [46]

disk under the consideration of pure rolling and pure sliding. These experiments were directly modeled using the proposed framework for the chosen material types, and the corresponding on-ground radial distributions were calculated. The comparison of the average radial distance from the disk center as obtained from the experiments, the numerical estimations reported in the original study, and the estimates obtained from the proposed framework with and without the consideration of Magnus lift forces have been shown in Fig. 10. It can be seen that for all numerical calculations, the distances are over-predicted when compared to the experimental data. Additionally, for most cases, our calculations compared well with the corresponding numerical results from the proposed study. The primary reason for the observed difference is that the calculations in the original study employed a con-
Table 2 Description of the different grain materials from the study reported in [3], which have been used for the simulations

\begin{tabular}{lll}
\hline Grain type & Density $\left(\mathrm{kg} / \mathrm{m}^{3}\right)$ & Friction coefficient \\
\hline AMS standard & 1819 & 0.4 \\
Kmag & 2542 & 0.5 \\
Coarse potash & 1910 & 0.6 \\
Limestone filler & 2604 & 0.4 \\
Triple 0-46-0 & 1834 & 0.5 \\
Muriate of potash & 1937 & 0.6 \\
Urea & 1257 & 0.4 \\
DAP & 1606 & 0.6 \\
Pell lime & 2105 & 0.4 \\
\hline
\end{tabular}

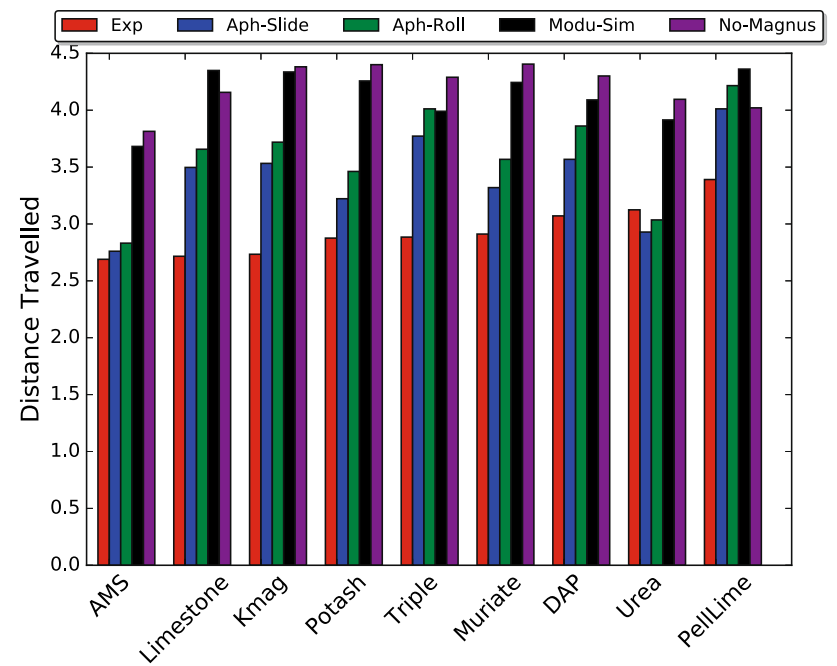

Fig. 10 Comparison of average radial distribution distance, for materials of differing densities and friction properties, as estimated from a the present model, without Magnus lift (No-Magnus), b present model, with Magnus lift (Modu-Sim), and c numerical simulations (Aph-Slide, and Aph-Roll) and $\mathbf{d}$ experimental data (Exp) reported by [3]

stant resistance coefficient for each particle-values ranging between $0.12 \mathrm{~m}^{-1}$ and $0.45 \mathrm{~m}^{-1}$ — while for our calculations, the drag coefficient was calculated based on a Reynolds number correlation, which leads to different values of the drag coefficient. Additionally, for further comparison of the overall trends, the radial distribution of particles around the disk was compared for each of the chosen materials. The distributions with and without lift forces have been presented in Fig. 11, and they compare very well with the reported trends of Gaussian-type distribution functions, with peak values approximately at $20 \%$ mass fraction located approximately at $4.5 \mathrm{~m}$. These numbers were specific to Boron micronutrient particles however, for which enough data was not available to perform a direct comparison. Hence, only qualitative comparison was achieved. 

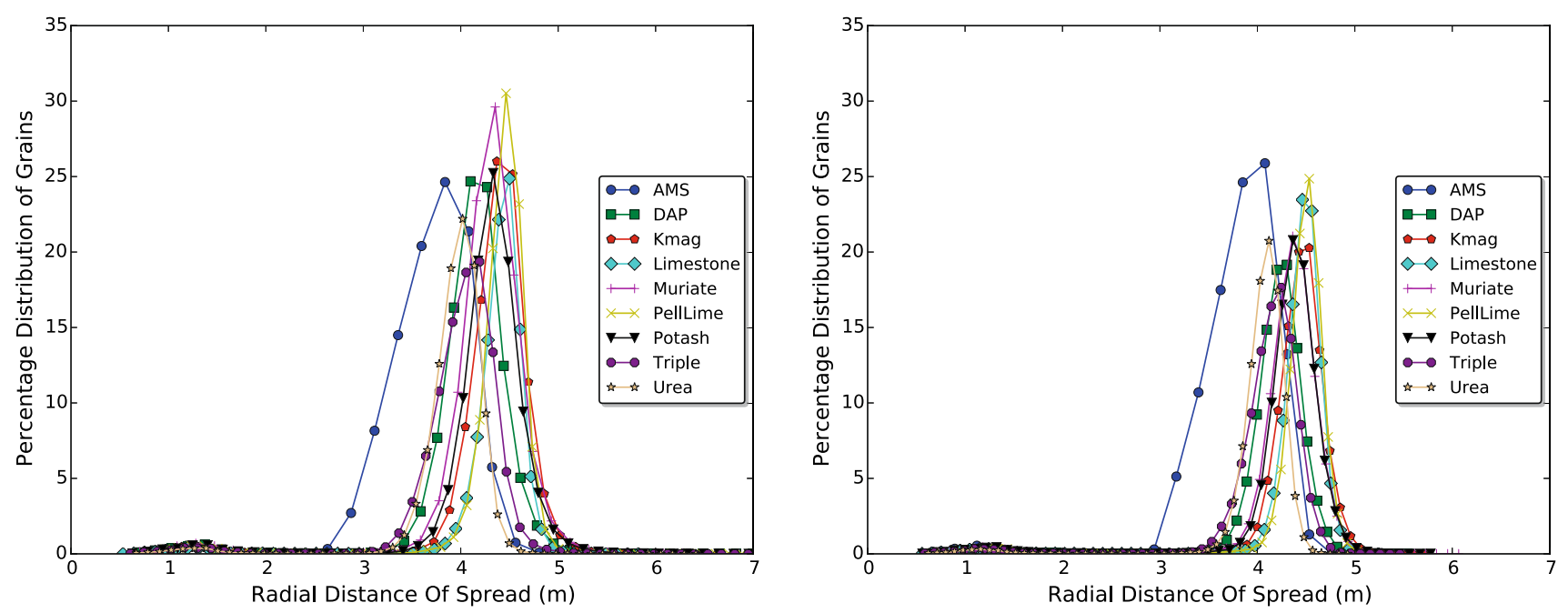

Fig. 11 Radial distribution patterns with and without considerations of Magnus lift (left and right, respectively) for various material types as listed in Table 2, which match the overall shape described in [3] very well

\section{Concluding remarks and outlook}

A modular DEM framework was developed for large-scale process simulations of industrial grain-handling systems. The mainstay of the developed framework was the existence of two distinct regimes of the particle dynamics, with distinctly separate time-scales and integration timestep requirements. This allowed a partitioning of the particle dynamics into two distinct modules. For the formulations discussed here, these modules comprised a contact-dominant and a sparsely interacting regime. Using appropriate integration schemes for each regime, with appropriately defined time-steps $\Delta t_{c}$ and $\Delta t_{f}$, and efficient data structure manipulations and simple parallelization techniques, we presented full process simulations of rotating spreaders with markedly large numbers of particles. As a basic estimation of the computational efficiency achieved here, using the simple estimates developed in Sect. 3.1 (see Eq. 49), and for $1.0 \mu \mathrm{s}$ as the contact duration time-step, and $1.0 \mathrm{~ms}$ being the inflight integration time-step with approximately 5 times the duration in flight, a direct efficiency of $C_{r} \approx 21 \%$ can be achieved. This is, of course, without any consideration of parallelization, and should only be taken as a baseline indicator of the proposed framework over a direct DEM simulation framework. It is noted here that some overhead due to data transfer, and data-structure manipulations, between the modules should be accounted for. While numerical examples of fertilizer spreader systems were extensively discussed, the framework is general enough to be employed in other similar applications as well, with little or no additional modifications. The extension of such a framework to such broader range of grain-handling processes is an area of continuing research. Additionally, while the translational velocities are reasonably well captured, the rotational dynamics pose notable concerns and require further research, both the issue of the accuracy of the angular velocity with ideal spheres as compared to real irregular particles and the Magnus force effect.

The observations made through the numerical experiments were also critical in developing valuable insights into the physical and numerical behavior of such systems. Specifically, we addressed the issue of a more generalized fluid-particle interaction model, with the incorporation of spin-induced (Magnus) lift. The effect of the lift was found to be significant with regards to both the individual particle trajectories, as well as the emergent distribution behavior. This aspect has not been extensively addressed in many numerical studies on spreaders. In fact, the most notable amongst the recent investigations discussing the role of lift was by [7], who reported substantial deviations in the particle trajectories, and emphasized on the relevance of further insights on this aspect. An additional consideration is the fact that inaccuracies in estimating the rotational velocities of the particles at the instant of launch-off may manifest themselves notably owing to the Magnus lift forces. Therefore, in combination with the fluid-particle interactions, care has to be taken to ensure that the rotational degrees of freedom of the individual particles are appropriately modeled.

Additionally, in comparison to existing literature on grainhandling systems governed by curved machine surfaces, the issue of mesh refinement and how geometric discretization errors propagate into the average particle distribution has been addressed in substantial detail. Phenomenologically, it was observed that for the individual particle trajectory, the discretization error leads to randomness in the contact forces, owing to the variations in the surface normal from element to element for a given coarse surface triangulation. On the average, this therefore leads to an effective enhancement of 
surface friction, which reduces with increasing refinement, as illustrated in Sect. 3.3.

The work presented here is part of an ongoing effort towards establishing large-scale DEM simulation frameworks for industrial grain-handling processes. The model developed, and the specific insights generated, are crucial for establishing the accuracy and robustness of DEM simulation frameworks, and for making such simulations more predictive.

Acknowledgments We would like to acknowledge the kindness of Dr. Van Liedekerke from the Ecole Normale Superieure and Dr. Coetzee from University of Stellenbosch, who responded promptly to all our enquiries regarding their work.

\section{Appendix 1: The case of a conical disk with no vanes}

Let us consider a conical disk of radius $R$ spinning around its vertical axis with angular velocity $\omega$. Let the Cartesian coordinate system $\{\mathbf{O}, \mathbf{i}, \mathbf{j}, \mathbf{k}\}$ be centered at the cone's vertex. The forces acting on the particle are

$\mathbf{f}_{g}=-g \mathbf{k}, \quad \mathbf{f}_{R}=f_{R} \mathbf{n}, \quad \mathbf{f}_{f}=\mu f_{R} \mathbf{n}_{\mathbf{v}}$.

The three forces above correspond to the weight, $\mathbf{f}_{g}$, the reaction against the cone, $\mathbf{f}_{R}$, and the friction resistance, $\mathbf{f}_{f}$. Furthermore, $\mathbf{n}$ is the inner normal to the cone at the particle's position, $\mathbf{n}_{\mathbf{v}}$ the normalized (when non-zero) relative velocity of the cone with respect to the particle and $f_{R}$ is the magnitude of the normal reaction to the particle's forces against the cone. Note that $f_{R}$ is a priori unknown but must be positive (no attractive forces). Consequently, the Second Law of Newton applied to the particle yields the following system:

$$
\begin{aligned}
& m \ddot{x}=f_{R}\left(\mu \mathbf{n}_{\mathbf{v}} \cdot \mathbf{i}-\sin \alpha \cos \theta\right) \\
& m \ddot{y}=f_{R}\left(\mu \mathbf{n}_{\mathbf{v}} \cdot \mathbf{j}-\sin \alpha \sin \theta\right) \\
& m \ddot{z}=f_{R}\left(\cos \alpha+\mu \mathbf{n}_{\mathbf{v}} \cdot \mathbf{k}\right)-m g,
\end{aligned}
$$

where $\theta$ is the standard polar angular coordinate and $\alpha$ the cone angle ( $\alpha=0$ for a flat disk). Since the particle is forced to move on the surface of the cone, the three coordinates $x$, $y$ and $z$ are linked by

$z=\tan \alpha \sqrt{x^{2}+y^{2}}$

which can readily be derived yielding

$\ddot{z}=\tan \alpha \frac{\left(x y^{2}+x^{3}\right) \ddot{x}+\left(x^{2} y+y^{3}\right) \ddot{y}+(y \dot{x}-x \dot{y})^{2}}{\left(x^{2}+y^{2}\right)^{3 / 2}}$

In order to be solved, this system of equations must be provided with initial conditions. Let us consider the following: $\mathbf{x}(0)=\left(\begin{array}{c}r_{0} \\ 0 \\ 0\end{array}\right), \quad \dot{\mathbf{x}}(0)=\left(\begin{array}{c}0 \\ v_{0} \\ 0\end{array}\right)$

where $r_{0} \in(0, R)$, with $R$ the radius of the disk.

Let us normalize this system of ODE's to obtain a nondimensional analog of it . By repeated application of direct substitution and the chain rule to the derivatives, one may obtain the following equivalent non-dimensional system:

$\ddot{\xi}_{i}=\phi_{R}\left(\mu \mathbf{n}_{\mathbf{v}} \cdot \mathbf{i}-\sin \alpha \cos \theta\right)$
$\ddot{\xi}_{j}=\phi_{R}\left(\mu \mathbf{n}_{\mathbf{v}} \cdot \mathbf{j}-\sin \alpha \sin \theta\right)$
$\ddot{\xi}_{k}=\phi_{R}\left(\cos \alpha+\mu \mathbf{n}_{\mathbf{v}} \cdot \mathbf{k}\right)-\gamma$

with

$\ddot{\xi}_{k}=\tan \alpha \frac{\left(\xi_{i} \xi_{j}^{2}+\xi_{i}^{3}\right) \ddot{\xi}_{i}+\left(\xi_{i}^{2} \xi_{j}+\xi_{j}^{3}\right) \ddot{\xi}_{j}+\left(\xi_{j} \dot{\xi}_{i}-\xi_{i} \dot{\xi}_{j}\right)^{2}}{\left(\xi_{i}^{2}+\xi_{j}^{2}\right)^{3 / 2}}$

and

$\xi(0)=\left(\begin{array}{c}\rho_{0} \\ 0 \\ 0\end{array}\right), \quad \dot{\xi}(0)=\left(\begin{array}{c}0 \\ v_{0} \\ 0\end{array}\right)$,

where the dot notation has been abused to refer to the derivatives with respect to the non-dimensional time

$\tau=t \omega$

or the units of time needed to complete a 1 radian turn by the spinning disk. The rest of the new non-dimensional parameters are

$\xi=\frac{1}{R}\left(\begin{array}{l}x \\ y \\ z\end{array}\right), \quad \phi_{R}=\frac{f_{R}}{m \omega^{2} R}, \quad \gamma=\frac{g}{\omega^{2} R}, \quad \rho_{0}=\frac{r_{0}}{R}$,

$\nu_{0}=\frac{v_{0}}{\omega R}$.

Thus distances have been normalized by the disk's radius and the forces by the modulus of the centripetal force felt by a particle stuck to the disk's rim edge. The variable $\gamma$ can be interpreted as a Froude's number for the system. From this form of the system it becomes apparent, for example, that the solution does not depend on the mass of the particle, since none of the known variables are defined in terms of it.

The system above can be solved numerically. In this work a simple forward Euler scheme has been employed, in which $\phi_{R}$ is taken from the old time-step in Eqs. 58 and subsequently updated using 59 . The rest of the parameter values are fixed to the quantities given in Table 3. 
Table 3 Values of the problem parameters used for the cone with no vanes benchmark

\begin{tabular}{ll}
\hline$\rho_{0}$ & 0.4 \\
$\nu_{0}$ & 0.02 \\
$\gamma$ & 0.0004 \\
$\alpha$ & $\pi / 12$ \\
\hline
\end{tabular}

\section{Appendix 2: Derivation of Eq. 19}

The incremental expression provided by Thornton reads:

$F_{t, \mathrm{el}}^{n}= \begin{cases}F_{t, \mathrm{el}}^{n-1}+k_{t}^{n} \Delta \delta_{t} & \text { if } \Delta F_{n} \geq 0 \\ F_{t, \mathrm{el}}^{n-1}\left(\frac{k_{t}^{n}}{k_{t}^{n-1}}\right)+k_{t}^{n} \Delta \delta_{t} & \text { if } \Delta F_{n}<0,\end{cases}$

where the super-index $n$ indicates the nth time-step and the operator $\Delta$ denotes the forward finite difference operator in time. It is direct to see that the first branch of 63 leads to the first branch of Eq. 19 by taking the limit when the time increments tend to 0. Similarly, the second branch becomes, after dividing it through by $k_{t}^{n}$

$\Delta\left(\frac{F_{t, \mathrm{el}}}{k_{t}}\right)=\Delta \delta_{t}$

which yields the expression of the second branch in 19 after dividing both sides by the time increment and taking the limit when it tends to 0 .

\section{References}

1. Adam S, Suzzi D, Radeke C, Khinast JG (2011) An integrated quality by design (qbd) approach towards design space definition of a blending unit operation by discrete element method (dem) simulation. Eur J Pharm Sci 42(1):106-115

2. Antypov D, Elliott JA (2011) On an analytical solution for the damped hertzian spring. EPL (Europhys Lett) 94(5):50004

3. Aphale A, Bolander N, Park J, Shaw L, Svec J, Wassgren C (2003) Granular fertiliser particle dynamics on and off a spinner spreader. Biosyst Eng 85(3):319-329

4. Cleary PW (1998) Predicting charge motion, power draw, segregation and wear in ball mills using discrete element methods. Miner Eng 11(11):1061-1080

5. Cleary PW (2001) Recent advances in dem modelling of tumbling mills. Miner Eng 14(10):1295-1319

6. Coetzee CJ, Lombard SG (2011) Discrete element method modelling of a centrifugal fertiliser spreader. Biosyst Eng 109(4):308325

7. Cool S, Pieters J, Mertens KC, Hijazi B, Vangeyte J (2014) A simulation of the influence of spinning on the ballistic flight of spherical fertiliser grains. Comput Electron Agric 105:121-131

8. Crowe CT, Schwarzkopf JD, Sommerfeld M, Tsuji Y (2011) Multiphase flows with droplets and particles. CRC press, Boca Raton

9. Dadvand P, Rossi R, Oñate E (2010) An object-oriented environment for developing finite element codes for multi-disciplinary applications. Arch Comput Methods Eng 17(3):253-297

10. Das S (2003) Physical aspects of process control in selective laser sintering of metals. Adv Eng Mater 5(10):701-711
11. Dennis SCR, Singh SN, Ingham DB (1980) The steady flow due to a rotating sphere at low and moderate reynolds numbers. J Fluid Mech 101(02):257-279

12. Dintwa E, Van Liedekerke P, Olieslagers R, Tijskens E, Ramon $\mathrm{H}$ (2004) Model for simulation of particle flow on a centrifugal fertiliser spreader. Biosyst Eng 87(4):407-415

13. Duran J (2012) Sands, powders, and grains: an introduction to the physics of granular materials. Springer, New York

14. Farazmand M, Haller G (2015) The maxey-riley equation: existence, uniqueness and regularity of solutions. Nonlinear Anal: Real World Appl 22:98-106

15. Feuillebois F, Lasek A (1978) On the rotational historic term in non-stationary stokes flow. Q J Mech Appl Math 31(4):435-443

16. Gouesbet G, Berlemont A (1999) Eulerian and lagrangian approaches for predicting the behaviour of discrete particles in turbulent flows. Prog Energ Combust Sci 25(2):133-159

17. Haider A, Levenspiel O (1989) Drag coefficient and terminal velocity of spherical and nonspherical particles. Powder Technol 58(1):63-70

18. Hofstee JW (1995) Handling and spreading of fertilizers: part 5, the spinning disc type fertilizer spreader. J Agric Eng Res 62(3):143162

19. Höhner D, Wirtz S, Kruggel-Emden H, Scherer V (2011) Comparison of the multi-sphere and polyhedral approach to simulate non-spherical particles within the discrete element method: Influence on temporal force evolution for multiple contacts. Powder Technol 208(3):643-656

20. Horner DA, Peters JF, Carrillo A (2001) Large scale discrete element modeling of vehicle-soil interaction. J Eng Mech 127(10): 1027-1032

21. Irazabal J (2015) Masters thesis: Numerical modelling of railway ballast using the discrete element method

22. Johnson KL (1987) Contact mechanics. Cambridge university press, Cambridge

23. Ketterhagen WR, am Ende MT, Hancock BC (2009) Process modeling in the pharmaceutical industry using the discrete element method. J Pharm Sci 98(2):442-470

24. Kim I, Elghobashi S, Sirignano WA (1998) On the equation for spherical-particle motion: effect of reynolds and acceleration numbers. J Fluid Mech 367:221-253

25. Kloss C, Goniva C, Hager A, Amberger S, Pirker S (2012) Models, algorithms and validation for opensource dem and cfd-dem. Prog Comput Fluid Dyn Int J 12(2-3):140-152

26. Kodam M, Curtis J, Hancock B, Wassgren C (2012) Discrete element method modeling of bi-convex pharmaceutical tablets: contact detection algorithms and validation. Chem Eng Sci 69(1):587601

27. Landau LD, Pitaevskii LP, Kosevich AM, Lifshitz M (1986) Theory of elasticity, 3rd edn., vol 7 (Course of theoretical physics), vol 1, 3rd edn. Butterworth-Heinemann,

28. Maxey MR, Riley JJ (1983) Equation of motion for a small rigid sphere in a nonuniform flow. Phys Fluids (1958-1988) 26(4):883889

29. Mishra BK (2003) A review of computer simulation of tumbling mills by the discrete element method: Part II-practical applications. Int J Miner Proc 71(1):95-112

30. Mishra BK (2003) A review of computer simulation of tumbling mills by the discrete element method: part I-contact mechanics. Int J Miner Proc 71(1):73-93

31. Morrison RD, Cleary PW (2004) Using dem to model ore breakage within a pilot scale sag mill. Miner Eng 17(11):1117-1124

32. Mukherjee D, Zohdi TI (2015) A discrete element based simulation framework to investigate particulate spray deposition processes. J Comput Phys 290:298-317 
33. Muzzio FJ, Shinbrot T, Glasser BJ (2002) Powder technology in the pharmaceutical industry: the need to catch up fast. Powder Technol 124(1):1-7

34. Oesterle B, Bui Dinh T (1998) Experiments on the lift of a spinning sphere in a range of intermediate reynolds numbers. Exp Fluids 25(1):16-22

35. O'Sullivan C, Bray JD (2004) Selecting a suitable time step for discrete element simulations that use the central difference time integration scheme. Eng Comput 21(2/3/4):278-303

36. Patterson DE, Reece AR (1962) The theory of the centrifugal distributor. I: motion on the disc, near-centre feed. J Agric Eng Res 7(3):232-240

37. Perret-Liaudet J, Rigaud E (2006) Response of an impacting hertzian contact to an order-2 subharmonic excitation: theory and experiments. J Sound Vib 296(1):319-333

38. Pöschel T, Schwager T (2005) Computational granular dynamics: models and algorithms. Springer, Berlin

39. Schwartz SR, Richardson DC, Michel P (2012) An implementation of the soft-sphere discrete element method in a high-performance parallel gravity tree-code. Granul Matter 14(3):363-380

40. Stronge WJ (2004) Impact mechanics. Cambridge university press, Cambridge

41. Thornton C, Cummins SJ, Cleary PW (2013) An investigation of the comparative behaviour of alternative contact force models during inelastic collisions. Powder Technol 233:30-46

42. Tijskens E, Ramon H, De Baerdemaeker J (2003) Discrete element modelling for process simulation in agriculture. J Sound Vib 266(3):493-514

43. Van Hinsberg M, ten Thije J, ten Thije Boonkkamp J, Clercx H (2011) An efficient, second order method for the approximation of the basset history force. J Comput Phys 230(4):1465-1478
44. Van Liedekerke P, Tijskens E, Dintwa E, Anthonis J, Ramon H (2006) A discrete element model for simulation of a spinning disc fertilizer spreader I. Single particle simulations. Powder Technol 170(2):71-85

45. Van Liedekerke P, Tijskens E, Dintwa E, Rioual F, Vangeyte J, Ramon H (2009) DEM simulations of the particle flow on a centrifugal fertilizer spreader. Powder Technol 190(3):348-360

46. Van Liedekerke P, Tijskens E, Ramon H (2009) Discrete element simulations of the influence of fertiliser physical properties on the spread pattern from spinning disc spreaders. Biosyst Eng 102(4):392-405

47. Van Liedekerke P, Piron E, Vangeyte J, Villette S, Ramon $\mathrm{H}$, Tijskens E (2008) Recent results of experimentation and DEM modeling of centrifugal fertilizer spreading. Granul Matter 10(4):247-255

48. Villette S, Cointault F, Piron E, Chopinet B (2005) Centrifugal spreading: an analytical model for the motion of fertiliser particles on a spinning disc. Biosyst Eng 92(2):157-164

49. Yan X, Gu P (1996) A review of rapid prototyping technologies and systems. Computer-Aided Des 28(4):307-318

50. Ye H, Liu XY, Hong H (2008) Fabrication of metal matrix composites by metal injection moldinga review. J Mater Proc Technol 200(1):12-24

51. Zhu HP, Zhou ZY, Yang RY, Yu AB (2008) Discrete particle simulation of particulate systems: a review of major applications and findings. Chem Eng Sci 63(23):5728-5770

52. Zohdi TI (2007) An introduction to modeling and simulation of particulate flows, vol 4. Siam, Philadelphia 